\title{
Video Article \\ Tracking the Mammary Architectural Features and Detecting Breast Cancer with Magnetic Resonance Diffusion Tensor Imaging
}

\author{
Noam Nissan ${ }^{1}$, Edna Furman-Haran ${ }^{2}$, Myra Feinberg-Shapiro ${ }^{3}$, Dov Grobgeld ${ }^{1}$, Erez Eyal ${ }^{1}$, Tania Zehavi ${ }^{4}$, Hadassa Degani $^{1}$ \\ ${ }^{1}$ Department of Biological Regulation, Weizmann Institute of Science \\ ${ }^{2}$ Unit of Biological Services, Weizmann Institute of Science \\ ${ }^{3}$ Department of Diagnostic Imaging, Meir Medical Center \\ ${ }^{4}$ Pathology Department, Meir Medical Center
}

Correspondence to: Hadassa Degani at hadassa.degani@weizmann.ac.il

URL: https://www.jove.com/video/52048

DOI: doi: $10.3791 / 52048$

Keywords: Medicine, Issue 94, Magnetic Resonance Imaging, breast, breast cancer, diagnosis, water diffusion, diffusion tensor imaging

Date Published: $12 / 15 / 2014$

Citation: Nissan, N., Furman-Haran, E., Feinberg-Shapiro, M., Grobgeld, D., Eyal, E., Zehavi, T., Degani, H. Tracking the Mammary Architectural Features and Detecting Breast Cancer with Magnetic Resonance Diffusion Tensor Imaging. J. Vis. Exp. (94), e52048, doi:10.3791/52048 (2014)

\section{Abstract}

Breast cancer is the most common cause of cancer among women worldwide. Early detection of breast cancer has a critical role in improving the quality of life and survival of breast cancer patients. In this paper a new approach for the detection of breast cancer is described, based on tracking the mammary architectural elements using diffusion tensor imaging (DTI).

The paper focuses on the scanning protocols and image processing algorithms and software that were designed to fit the diffusion properties of the mammary fibroglandular tissue and its changes during malignant transformation. The final output yields pixel by pixel vector maps that track the architecture of the entire mammary ductal glandular trees and parametric maps of the diffusion tensor coefficients and anisotropy indices.

The efficiency of the method to detect breast cancer was tested by scanning women volunteers including 68 patients with breast cancer confirmed by histopathology findings. Regions with cancer cells exhibited a marked reduction in the diffusion coefficients and in the maximal anisotropy index as compared to the normal breast tissue, providing an intrinsic contrast for delineating the boundaries of malignant growth. Overall, the sensitivity of the DTI parameters to detect breast cancer was found to be high, particularly in dense breasts, and comparable to the current standard breast MRI method that requires injection of a contrast agent. Thus, this method offers a completely non-invasive, safe and sensitive tool for breast cancer detection.

\section{Video Link}

The video component of this article can be found at https://www.jove.com/video/52048/

\section{Introduction}

Breast cancer is the most common cause of cancer among women worldwide. Early detection of breast cancer has a critical role in improving the quality of life and survival of breast cancer patients. The current standard methods for breast cancer detection are based on $\mathrm{x}$-ray mammography and ultrasound scanning. The insufficient sensitivity and specificity of these techniques, particularly for detecting lesions in dense breasts, has stimulated the development of other techniques including breast magnetic resonance imaging (MRI). Dynamic contrast enhanced (DCE) MRI has been established as a powerful tool for the detection and diagnosis of breast cancer ${ }^{1,2}$ and is frequently facilitated by computer aided diagnosis means ${ }^{3}$. Currently it is used for special cases, such as high risk patients ${ }^{4}$, but not for routine screening, presumably because of the high costs, the need to use an injection of a contrast agent, the lack of standardization and the variable specificity in differentiating benign from

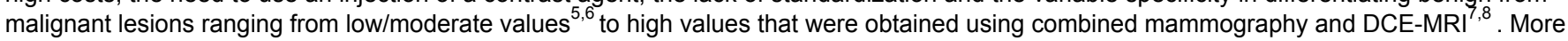
recently, diffusion weighted MRI and the resulting maps of apparent diffusion coefficient (ADC) have been evaluated as a complement method to DCE-MRI and it was shown that ADC values can help distinguish between cancers, benign lesions and normal breast tissue ${ }^{9,10}$. In addition, studies of breast diffusion tensor imaging (DTI) were initiated in healthy volunteers and patients with breast lesions at field strength of $1.5 \mathrm{~T}^{11-15}$ and of $3 T^{16-24}$. Most of these studies reported ADC and fractional anisotropy (FA) values ${ }^{11,12,14,15,20-23}$ and found these two parameters to be reproducible with $A D C$ values more reproducible than $F A^{13,20}$. The results of these studies indicated that malignant lesions exhibit low ADC values as compared to normal tissue and benign lesions, however, conflicting results were reported on the values and diagnostic capability of $\mathrm{FA}^{11,12,14,20-23}$. In a set of 3 T- DTI studies the values of the three tensor eigenvalues and eigenvectors in the breast tissue frame were reported as well, and the results were presented in vector maps of the main eigenvector and parametric maps of the eigenvalues, ADC, FA and a maximal anisotropy index ${ }^{16-19,24}$. In these studies the main diffusion eigenvalue and the maximal anisotropy were shown to serve as the most sensitive independent parameters for the detection and diagnosis of cancer lesions

The breast is composed of fibroglandular tissue and fat tissue. The fibroglandular tissue is further composed of many lobes, which are highly variable in size and shape. Each lobe microstructure includes the functional mammary tree and associated lobules forming the glandular tissue, and the surrounding connective-fibrous tissue. Most mammary malignancies start by aberrant proliferation of epithelial cells in the ducts or 
lobules, developing in situ carcinoma, which by infiltration into the surrounding tissue turn into invasive carcinoma. Therefore, the ductal/lobular structures are an imperative area of investigation of malignant breast transformation.

The structural features of the ductal trees were first investigated ex vivo in 1840 by Sir Astley Cooper using injection of colored wax to the ducts of mastectomy specimens ${ }^{25}$. Recently, computer derived tracking of whole-breast ductal trees has been achieved in few human breasts using mastectomy specimens ${ }^{26,27}$. The work presented here shows that parameters obtained by in vivo diffusion tensor imaging provide information associated with the distinct mammary tissue microstructural features, enabling also non-invasive breast cancer detection.

The physical principles underlying breast diffusion tensor imaging are based on MRI capability to measure and quantify anisotropic water diffusion in restricted environments ${ }^{28}$. In general, water diffusion in homogeneous solutions is free and isotropic, however, if the water movement is halted because of restriction by impermeable walls the diffusion becomes anisotropic with a fast free diffusion parallel to the walls and a slower restricted diffusion perpendicular to the walls (Figure 1). Water diffusion in tissues is complex and depends on structural and physiological features of the intra- and extracellular compartments including cells' sizes, cells' density, extracellular tortuosity and water exchange through membranes, as well as on the presence of vascular and lymphatic networks (Figure 2).

\section{Isotropic-Free Diffusion}
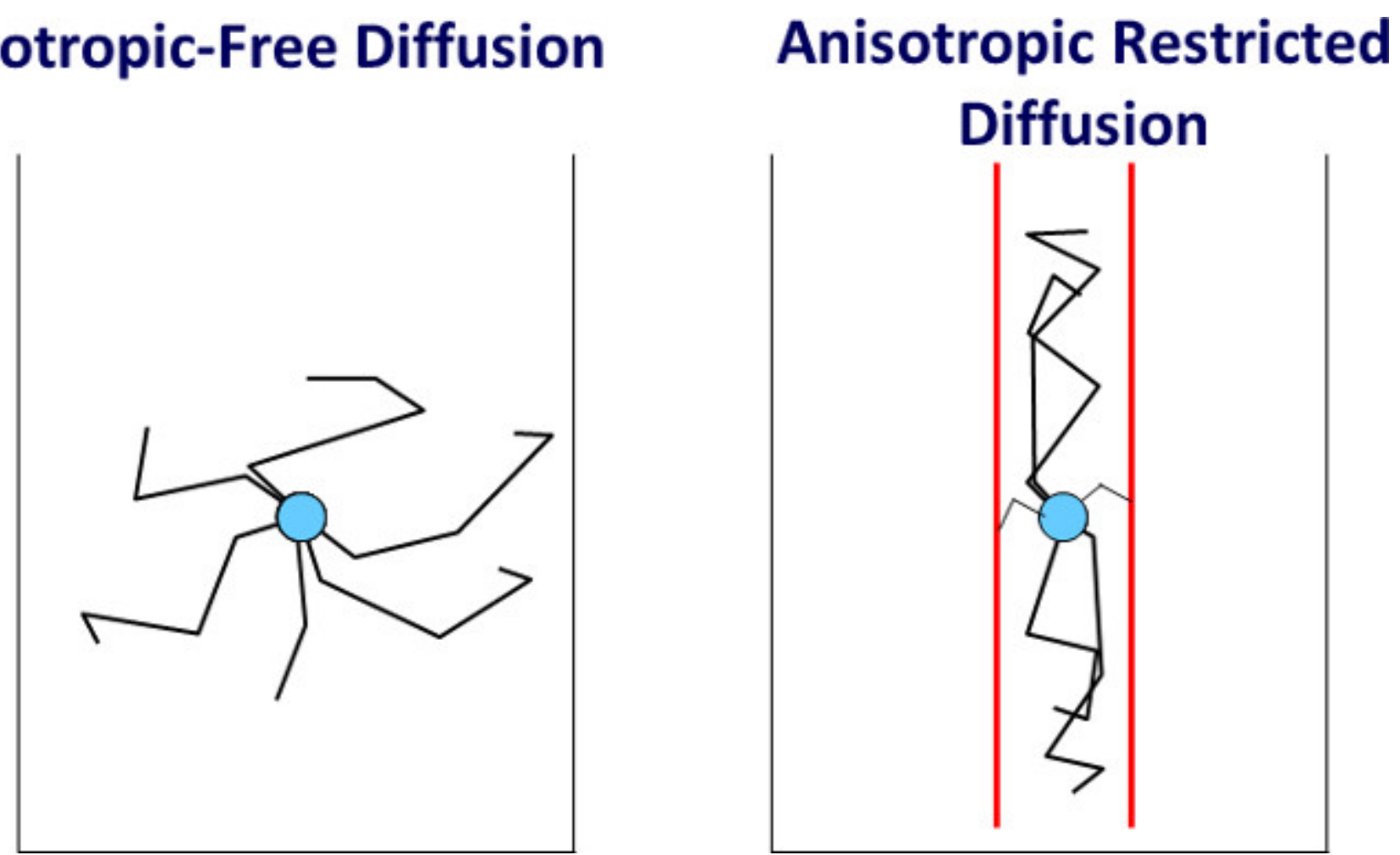

Figure 1: Free and restricted diffusion. Schematic drawing of a water molecule free diffusion (left) and diffusion restricted by impermeable walls (right). 


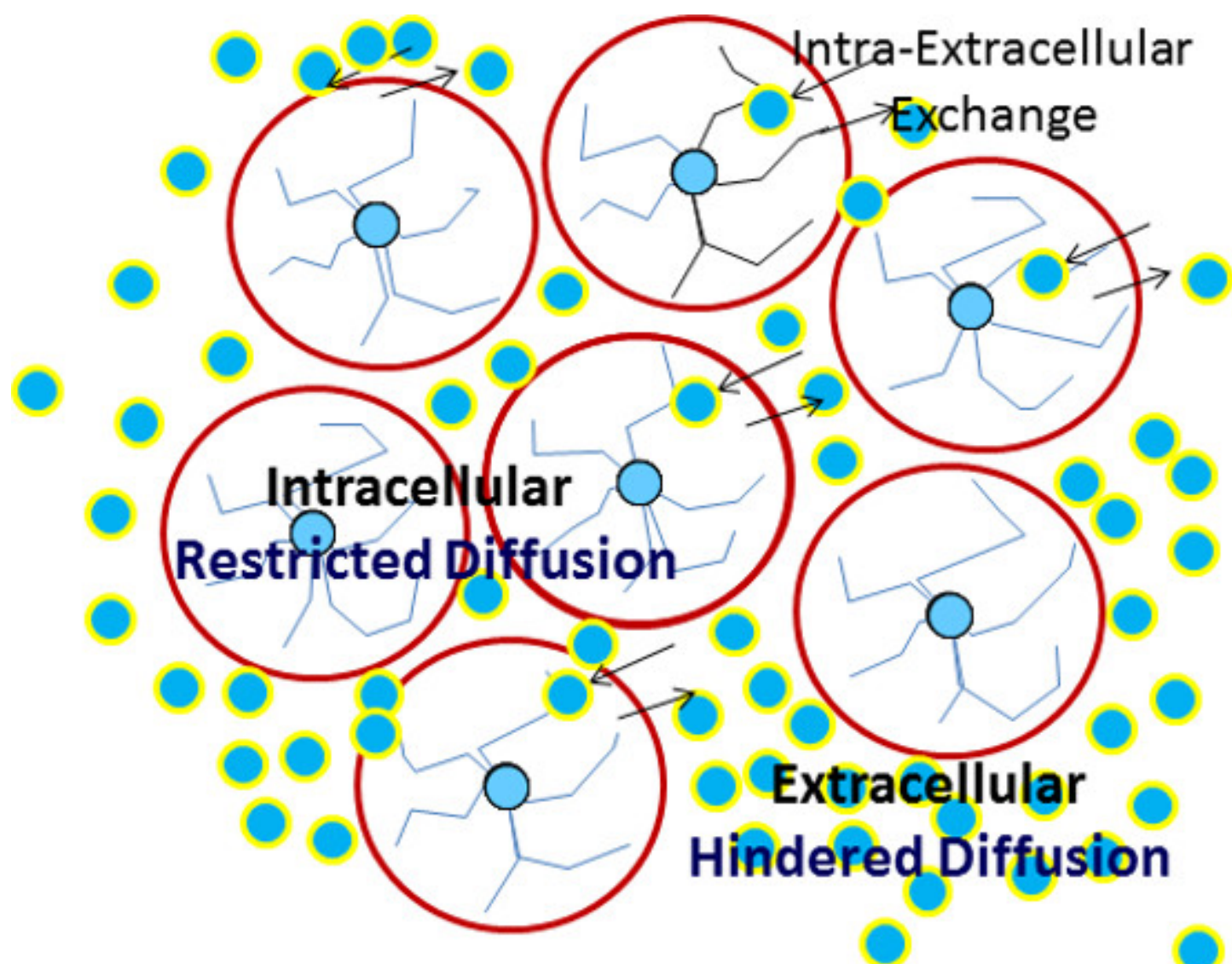

Figure 2: Complex diffusion in a tissue. Schematic drawing of water diffusion in a cellular system showing water molecules movement in the extracellular and intracellular compartments and water exchange (arrows) between these two compartments.

Due to the specific architectural features of the breast the diffusion of water molecules in the mammary ducts and lobules present a particular example of restricted and anisotropic movement: In parallel to the walls of the ducts and lobules the diffusion is close to that of free diffusion but in the directions perpendicular to the walls it is restricted by the walls, composed of two layers of cell and basement membrane. Consequently the diffusion in the ductal/glandular system is relatively fast and anisotropic. On the other hand, the diffusion in the connective fibrous tissue surrounding the ducts is fast and isotropic as a result of the high water content and low cell density in this tissue (Figures $\mathbf{3}$ and $\mathbf{4}$ ). In the presence of malignancy, blockage of the ducts and lobules by cancer cells increases the tortuosity and restriction of the water movement, causing a reduction in the diffusion coefficients in all directions and in the anisotropic movement (Figure 3 and 4). 

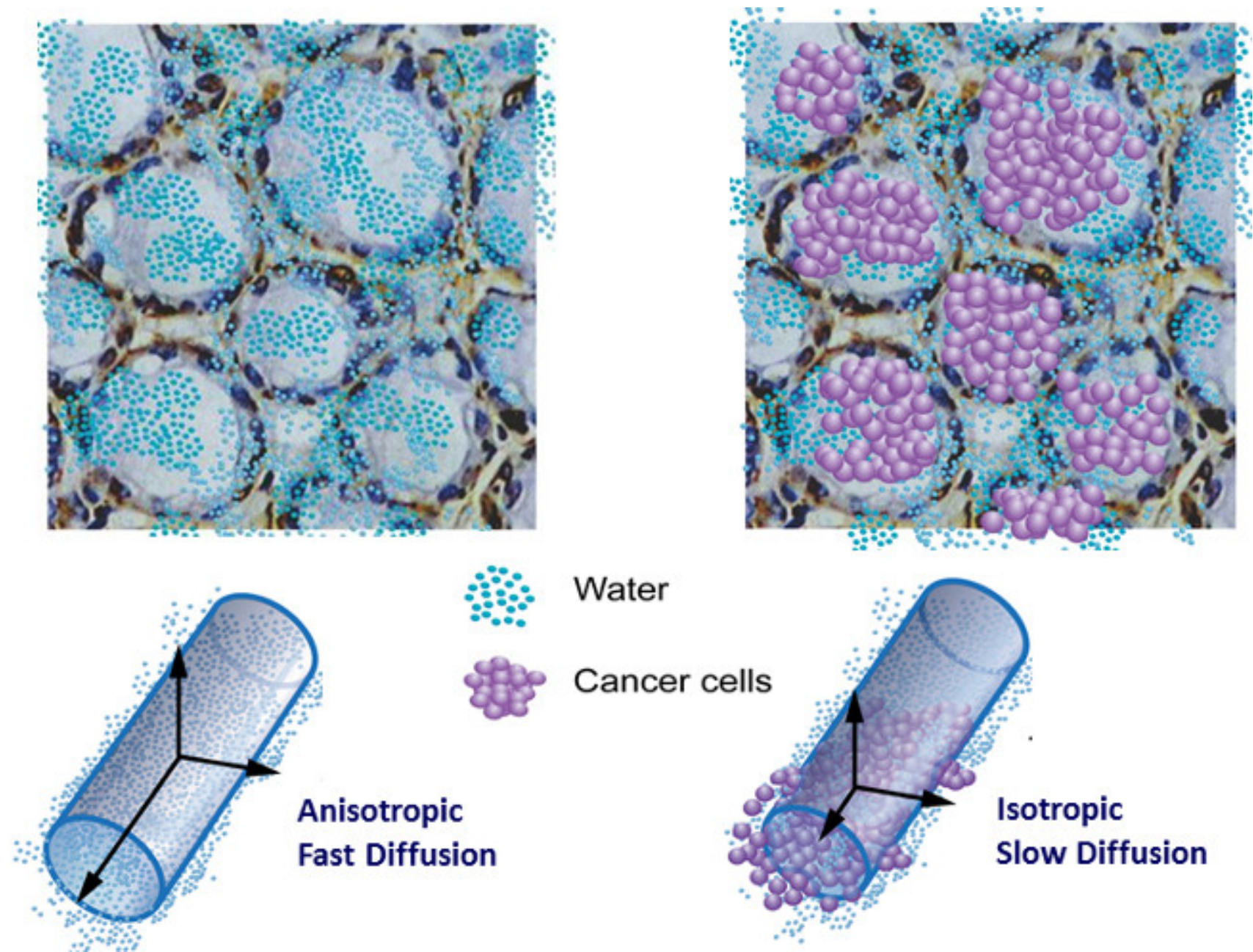

Figure 3: Diffusion in breast lobules. Schematic drawing of a cut through the lobules and the water diffusion inside one lobule. Left: diffusion of water restricted by the lobules' walls showing fast diffusion parallel to the walls and restricted diffusion perpendicular to the walls. Right: diffusion in lobules with cancer cells. The diffusion in the extracellular compartment is highly hindered but similar in all directions and hence, nearly isotropic.
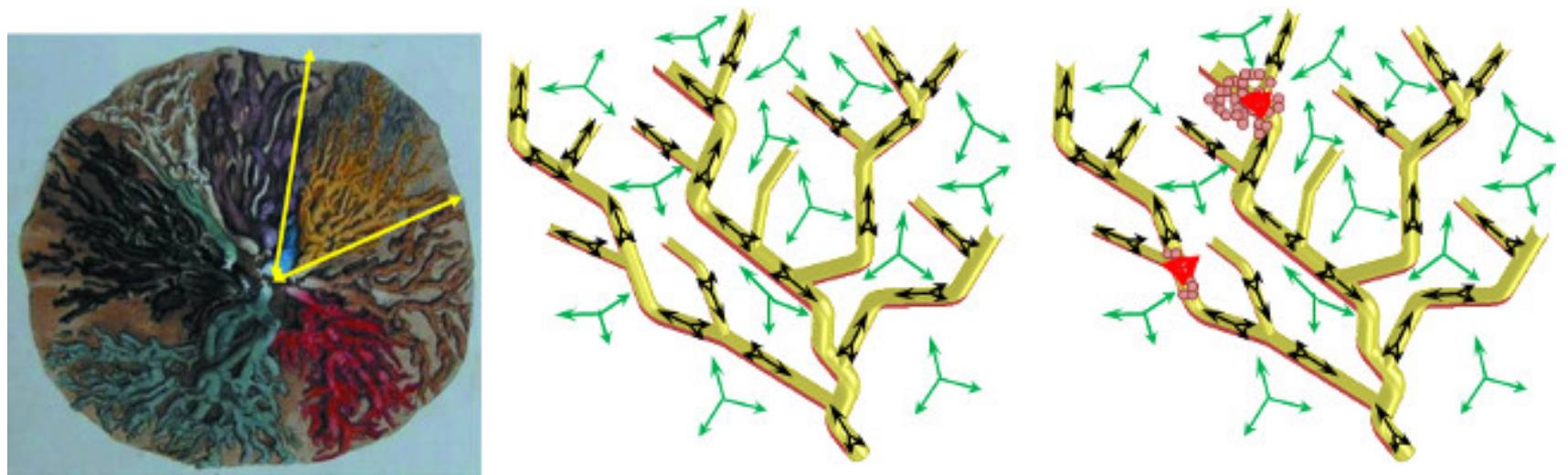

Figure 4: Water diffusion in the ductal tree system. Left: Mammary ducts injected with colored wax, showing their radiated direction, and their inter-ramification ${ }^{25}$. Middle: Schematic drawing of a normal ductal tree with vectors indicating the diffusion inside the ducts (black arrows) and in the connective tissue (green arrows). Right: Schematic drawing of a ductal tree with two loci of cancer cells (purple). Red arrows exhibit the diffusion in the cancers.

This paper describes in detail the diffusion tensor scanning method and the processing algorithms and software analysis of the DTI datasets that enabled detecting breast malignancy. All cancers were confirmed by histopathology findings of breast biopsy and/or surgical specimens. We also describe the T2 weighted scanning protocol for obtaining the breast anatomical features, as well as the DCE scanning protocol that served as a reference method for evaluating the DTI detection sensitivity. Please click here to view a larger version of this figure. 


\section{Protocol}

NOTE: All protocols were approved by the Internal Review Board of Meir Medical Center, Kfar-Saba, Israel and a signed informed consent was obtained from all subjects.

\section{Patient Preparation and Positioning in the MRI Scanner}

1. Insert an intravenous catheter in the left or right arm for contrast agent injection.

2. Ensure that the patient is lying in the prone position with both breasts hanging freely in the bilateral openings of the breast coil as demonstrated in Figure 5.

3. Ensure that the head is positioned on a pillow for patient's head and neck comfort and both arms are placed above the head as demonstrated in Figure 5.

4. Ensure that each breast is in a central positioning, hanging loose and as deeply as possible within the respective coil opening as demonstrated in Figure $\mathbf{5}$.

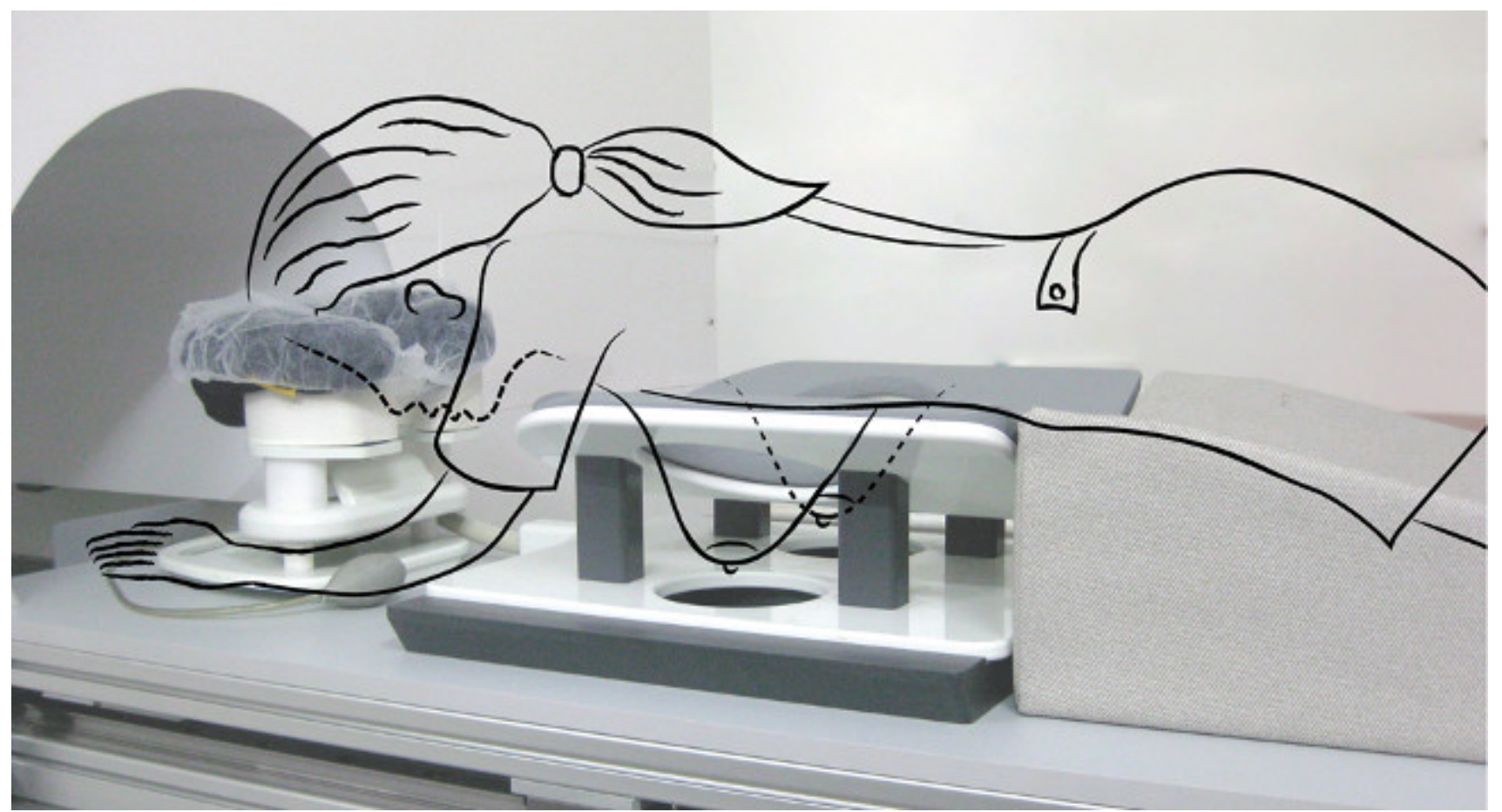

Figure 5: Positioning of a woman for breast MRI scanning. Note the prone position and the free hanging of both breasts in the bilateral openings of the breast coil.

5. Connect the automated injection pump to the intravenous catheter.

\section{MRI Scanning}

1. Acquire with the MRI scanner a pilot image for localizing both breasts and determine the field of view (FOV), number of slices and slice thickness to completely cover both breasts with extension to the axilla and to the chest wall.

2. In all the following scanning sequences performed with the MRI scanner, keep the same value of the FOV, slice number and slice thickness for each case. For example, acquire 60 axial slices with FOV of $360 \mathrm{~mm}$ in the read and phase direction, slice thickness ranging between 2 to $3 \mathrm{~mm}$, depending on the size of the breasts, and a phase encoding direction $L>>R$ or $R>$ s. Vary the in-plane resolution in the following sequences depending on the acquisition matrix dimension used for each sequence as indicated in Table 1.

\begin{tabular}{|l|l|l|l|l|}
\hline Experimental parameter & 2D T2 weighted & 2D DTI & 2D GE Field mapping & 3D DCE \\
\hline Repetition time (TR) msec & 5,500 & 10,800 & 669 & 6.8 \\
\hline Echo time (TE) msec & 122 & 120 & 4.92 & 2.49 \\
\hline TE(2) & & & 7.38 & \\
\hline Flip angle, degrees & & & 60 & 18 \\
\hline Acquisition matrix & $640 \times 428$ & $192 \times 192$ & $64 \times 192$ & $428 \times 428$ \\
\hline Averages & 1 & 1 & 1 & 1 \\
\hline
\end{tabular}




\begin{tabular}{|l|l|l|l|l|}
\hline concatenations & 4 & 1 & 1 & 1 \\
\hline Turbo factor & 21 & & & \\
\hline Number of trains per slice & 11 & & & \\
\hline Echo spacing, msec & 10 & 0.79 & & \\
\hline Bandwidth, Hz/pixel & 300 & 1860 & 1532 & 560 \\
\hline $\begin{array}{l}\text { In plane scanning } \\
\text { resolution }\end{array}$ & $0.56 \times 0.84$ & $1.9 \times 1.9$ & & $0.8 \times 0.8$ \\
\hline In plane image resolution & $0.56 \times 0.56$ & $1.9 \times 1.9$ & $1.9 \times 1.9$ & $0.8 \times 0.8$ \\
\hline Acquisition time, min:sec & $4: 26$ & $6: 09$ & $1: 28$ & $1: 06(\times 9)$ \\
\hline \# of diffusion gradients & & 30 & & \\
\hline Fat suppression & no & FAT-SAT / SPAIR ${ }^{1}$ & & no \\
\hline b-values, sec/mm & & 0,700 & & \\
\hline
\end{tabular}

Table 1: Experimental parameters of the sequences used in this study. ${ }^{1}$ FAT-SAT: Fat Saturation. SPAIR: Spectral Attenuated Inversion Recovery.

3. Localize with the user interface computer of the MRI scanner a region that includes both breasts and the axilla and define a shimming box. Apply an iterative shimming strategy to optimize the magnetic field ${ }^{29}$.

1. Display the proton spectrum on the screen of the user interface computer and adjust the shimming by centering the frequency on the water resonance frequency and then on the fat resonance frequency. Repeat the shimming until it best separates the fat and water signals and optimizes maximum signal intensity and shape as revealed in the proton spectrum. Verify that the irradiation frequency is centered on the water resonance frequency.

4. Apply through the user interface computer a 2D transversal T2-weighted turbo spin-echo multi slice sequence at high spatial resolution without fat saturation using generalized auto-calibrating partially parallel acquisition (GRAPPA), with acceleration factor 2 . Fix the parameters that will pop up on the screen according to their values in the column "2D T2 weighted" in Table 1.

5. Apply through the user interface computer a 2D transversal, fat suppressed, spin-echo diffusion tensor imaging - DTI using the twice refocused echo planar imaging sequence ${ }^{30}$, using GRAPPA, with acceleration factor 2 . Fix the parameters that will pop up on the screen according to their values in the column "2D DTI" in Table 1.

6. Apply through the user interface computer a field mapping sequence for correcting geometric distortions in echo-planar imaging (EPI), and obtain phase differential images as described by Jezzard and Balaban ${ }^{31}$. Ensure that the sequence includes acquisition of $2 \mathrm{D}$ transversal gradient echo (GE) images with two different in-phase echo times, and that the phase encoding direction is the same as in the DTI sequence (step 2.5). Fix the parameters that will pop up on the screen according to their values in the column "2D GE Field mapping" in Table 1.

7. Apply through the user interface computer a dynamic contrast enhanced protocol using a 3D fast gradient echo sequence without fatsuppression, with parameters optimized according to the three time point (3TP) method ${ }^{32}$. Fix the parameters that will pop up on the screen according to their values in the column "3D DCE" in Table 1.

1. Record two initial precontrast images according to the protocol in step 2.7.

8. Inject $15 \mathrm{sec}$ before the end of the second pre-contrast acquisition the contrast agent gadopentetatedimeglumine, at a dose of $0.1 \mathrm{mmol} / \mathrm{kg}$ body weight, using an automated pump at a rate of $2 \mathrm{ml} / \mathrm{sec}$, followed by $20 \mathrm{ml}$ of saline flush, at $2 \mathrm{ml} / \mathrm{sec}$.

1. Continue recording seven sequential 3D datasets according to the protocol in step 2.7 at $54,120,186,252,318,384$, and 450 sec post contrast injection.

9. Apply through the user interface computer a 2D transversal T2-weighted fat suppressed turbo spin-echo multi slice sequence at high spatial resolution using GRAPPA, with acceleration factor 2 . Use the experimental details similar to those used for T2 weighted imaging described in step 2.4 . NOTE: A time course summarizing the steps in the MRI protocol is provided in Figure 6.

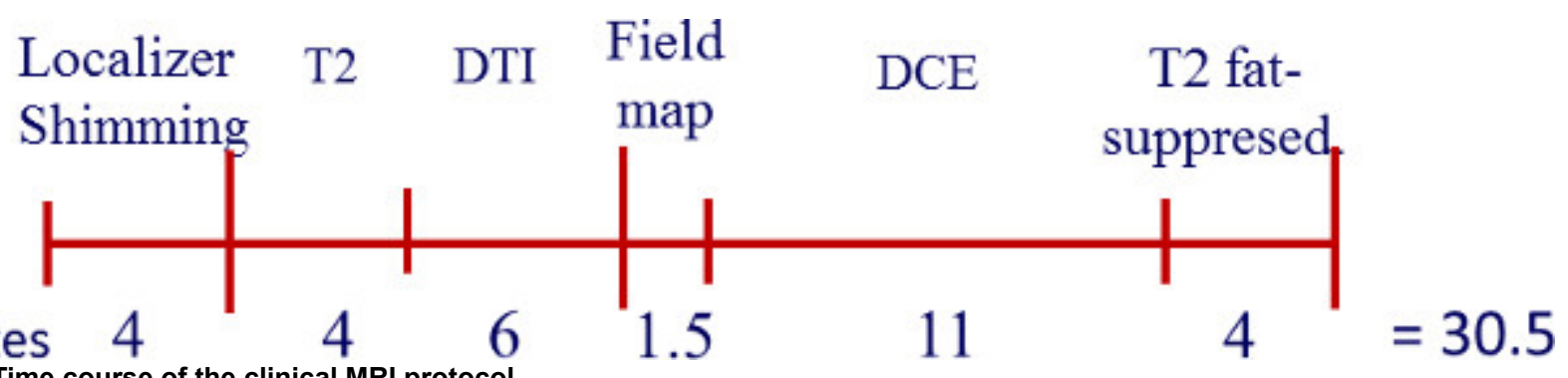

$\underset{\text { Figure }}{\operatorname{minu}}$ : Time course of the clinical MRI protocol. 


\section{Image Processing}

1. Transfer the entire dataset obtained by all the scanning sequences (described in steps $\mathbf{2 . 4} \mathbf{- 2 . 9}$ ) to a remote workstation or personal computer and process the entire datasets of images with software programs devoted to analyze breast diffusion tensor imaging and DCEMRI.

1. Here, perform the steps described below using the homebuilt software package written in $\mathrm{C}++$ that was verified by using the programing environment of MATLAB ${ }^{19}$. In general, commercial scanners provide image processing tools for brain DTI experiments that can be adapted for the breast but may not have all the processing functions applied by our homebuilt software package

2. Perform Image processing of the DTI datasets using a dedicated DTI image processing software following the steps in the flowchart of Figure 7.

\section{MRI protocol for DTI}

Acquire 2D axial fast $\mathrm{T} 2$ weighted images; Acquire diffusion weighted $2 \mathrm{D}$ axial images for DTI using echo-planar imaging (EPI).

\section{Computer-Loading}

Transfer to a work station all the datasets obtained with the MRI protocols

\section{Computer-Fitting}

Calculate pixel by pixel the diffusion coefficient in each direction according to the Stejskal-Tanner equation and apply a non linear best fit regression algorithm to calculate the rank-2 symmetric diffusion tensor

\section{Computer- apply principle component analysis}

For each pixel, diagonalize the diffusion tensor to yield three eigenvalues $\lambda_{1}, \lambda_{2}, \lambda_{3}$ and their corresponding eigenvectors $v_{1}, v_{2}, v_{3}$

\section{Computer- parametricmaps}

Display the 2D images, through the entire breasts, of $\lambda_{1}$ and also $\lambda_{2}$ and $\lambda_{3}$ and their combinations such as $\lambda_{1}-\lambda_{3}$ and $\left(\lambda_{1}+\lambda_{2}+\lambda_{3}\right) / 3$

\section{Computer- diffusion direction and ductal tracking}

Display 2D vector map of the prime eigenvector, $v_{1}$, and a color coded map of the main direction of this vector

Figure 7: Flowchart of the steps involved in DTI processing.

1. Use the DTI software program to evaluate the noise level outside of the breast and the remaining tissue in 3 to 4 regions of interest (of approximately $1 \mathrm{~cm}^{2}$ ) and find the maximum noise level.

2. Use the DTI software program to calculate pixel by pixel, in all pixels above the maximum noise level and in each of the 60 slices, the six diffusion coefficients, $D_{x x}, D_{y y}, D_{z z}, D_{x y}, D_{x z}, D_{y z}$, of the symmetric tensor $D$ described below ${ }^{33}$ (see Figure 8)

$$
\underline{D}=\left\{\begin{array}{ccc}
D_{x x} & D_{x y} & D_{x z} \\
D_{y x} & D_{y y} & D_{y z} \\
D_{z x} & D_{z y} & D_{z z}
\end{array}\right\}
$$

Where $D_{x y}=D_{y x}, D_{z y}=D_{y z}, D_{z x}=D_{x z}$.

1. In order to calculate the tensor components use a non-linear regression fitting program based on the Stejskal-Tanner equation: $\mathrm{Si}, \mathrm{j}(\mathrm{b})=\mathrm{Si}(0) \exp (-\mathrm{bD})$, where $\mathrm{Si}, \mathrm{j}(\mathrm{b})$ is the signal intensity of pixel $\mathrm{i}$ with a diffusion gradient in the direction $\mathrm{j}$ and $\mathrm{Si}(0)$ is the signal 
intensity of the same pixel at $b=0 \mathrm{~mm}^{2} \cdot \mathrm{s}$. This fitting minimized the sum of the squares of the 30 nonlinear functions for each diffusion gradient direction in the six diffusion tensor variable components by a modification of the Levenberg-Marquardt algorithm ${ }^{34}$.This step was performed by the free software cminpack (http://devernay.free.fr/hacks/cminpack/index.html).

\section{$\mathrm{b}=0 \mathrm{~s} / \mathrm{mm}^{2}$}

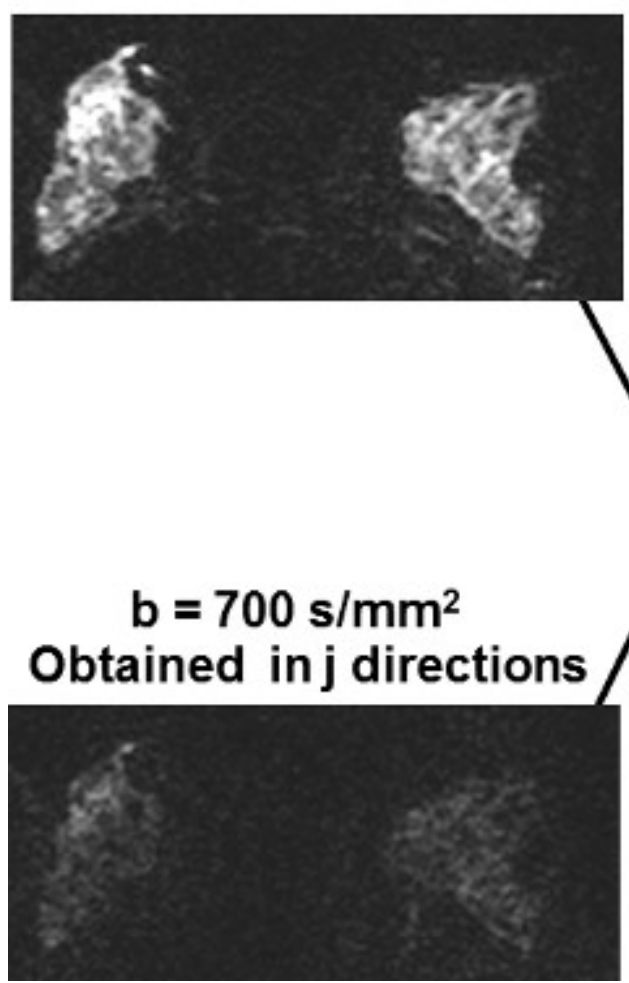

\section{Symmetric}

\section{Diffusion Tensor}

$\underset{\begin{array}{c}\text { Non Linear } \\ \text { Regression }\end{array}}{\stackrel{S_{\mathrm{ij}} / \mathrm{S}_{\mathrm{i}}(0)=\exp \left(-b D_{i j}\right)}{\longrightarrow}} \quad \underline{D}=\left\{\begin{array}{lll}D_{x x} & D_{x y} & D_{x z} \\ D_{y x} & D_{y y} & D_{y z} \\ D_{z x} & D_{z y} & D_{z z}\end{array}\right\}$

$$
D_{y x}=D_{x y} ; D_{z y}=D_{y z} ; D_{z x}=D_{x z}
$$

Figure 8: Schematic drawing of the calculation steps yielding the symmetric diffusion tensor.

3. Use the DTI software to diagonalize the symmetric diffusion tensor in each pixel by applying principal component analysis (PCA) ${ }^{35}$. NOTE: PCA is a commonly used method to reduce the dimensionality of the data and find a linear transformation that maps the correlated pixel parameters into a new coordinate system of uncorrelated and orthogonal axes. This process yields for each pixel three eigenvectors $\left(v_{1}\right.$, $v_{2}, v_{3}$ ), defining the diffusion direction in three orthogonal axes of an ellipsoid shape that coincides with the diffusion frame of the tissue, and the corresponding three diffusion eigenvalues, arranged from high to low, that determine three directional diffusion coefficients $\left(\lambda_{1}, \lambda_{2}, \lambda_{3}\right)$ (see Figures 9 and 10). 


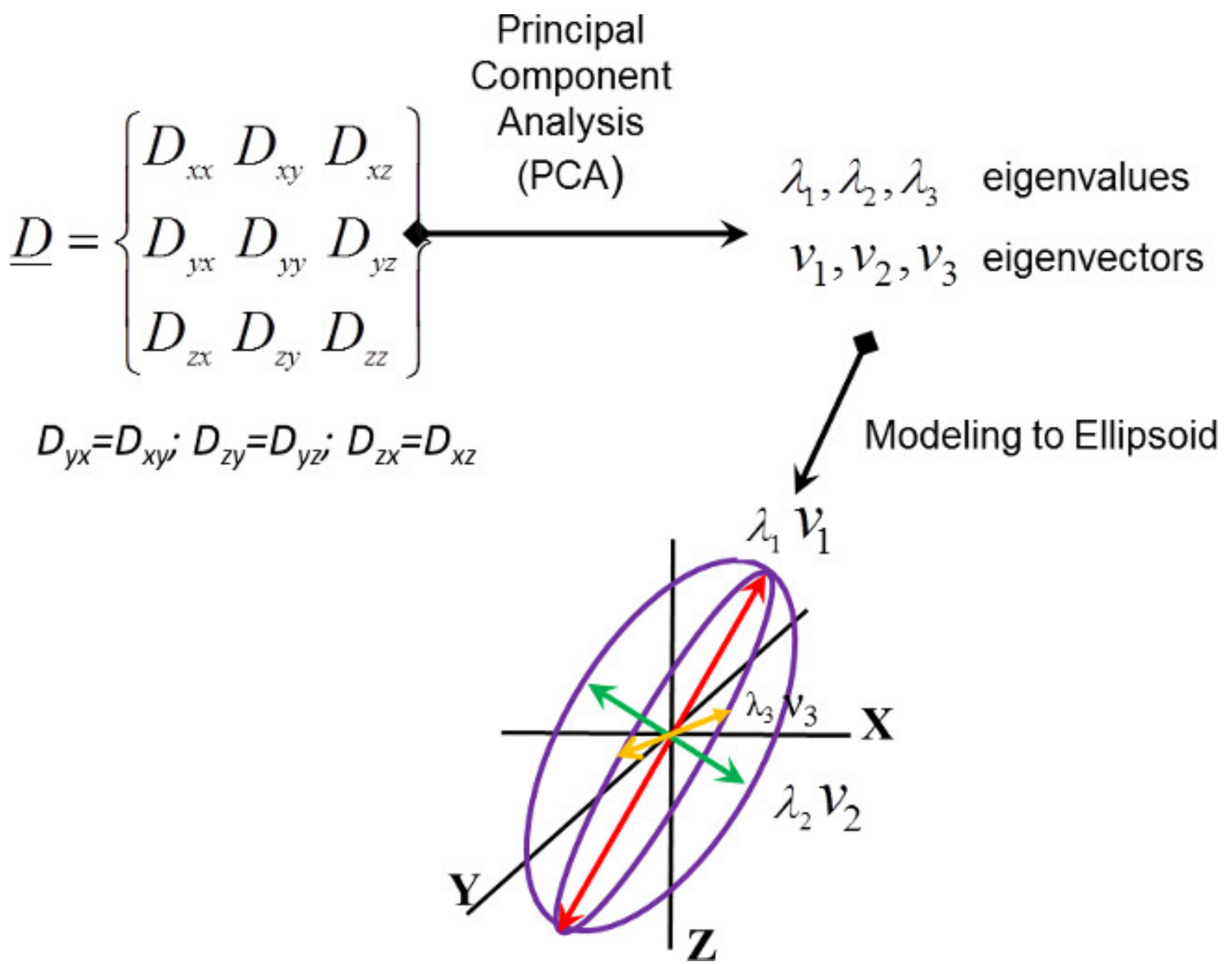

Figure 9: Schematic drawing of the calculation steps yielding the eigenvalues and eigenvectors coinciding with the diffusion frame of the tissue and the modeling to ellipsoid. 


\section{Diffusion in a duct}

\section{water molecules}

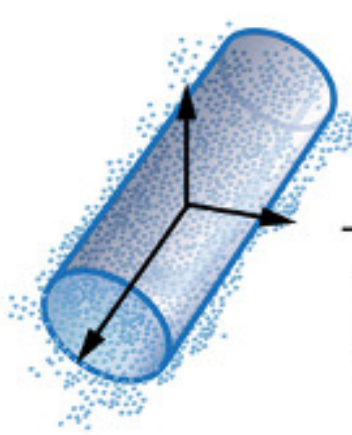

\section{Fraction of signal intensity \\ change in $\mathrm{N}$ directions}

Output of eigenvectors (v)

and corresponding eigenvalues $(\lambda)$
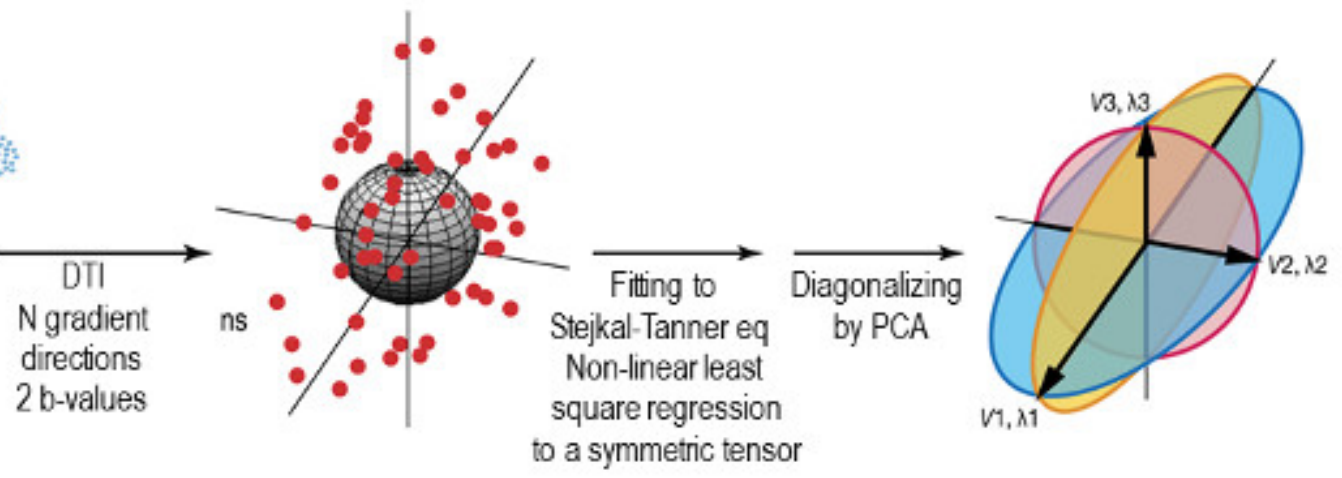

Diffusion in a duct

blocked by cancer
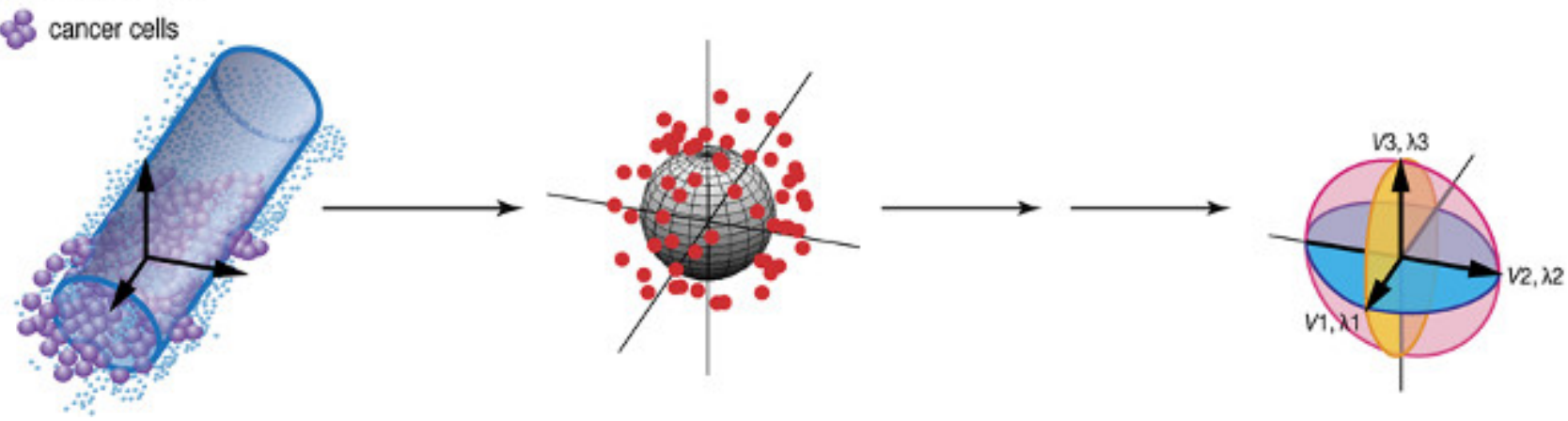

Figure 10: Schematic drawing of the main processing steps that calculate the diffusion eigenvectors and eigenvalues in normal ductal tissue and in cancer tissue.

4. Use the DTI software program to calculate for each pixel the apparent diffusion coefficient (ADC) defined as the average of the three eigenvalues $\langle\lambda\rangle=\left(\lambda_{1}+\lambda_{2}+\lambda_{3}\right) / 3$.

5. Use the DTI software program to calculate for each pixel the maximal absolute anisotropy index defined as the difference $\left(\lambda_{1}-\lambda_{3}\right)$.

6. Use the DTI software program to calculate for each pixel the fractional anisotropy (FA) index that ranges between 0 (isotropic diffusion) to 1 (free diffusion in one direction) according to the following equation:

$$
F A=\frac{\sqrt{3\left[\left(\lambda_{1}-\langle\lambda\rangle\right)^{2}+\left(\lambda_{2}-\langle\lambda\rangle\right)^{2}+\left(\lambda_{3}-\langle\lambda\rangle\right)^{2}\right]}}{\sqrt{2\left(\lambda_{1}{ }^{2}+\lambda_{2}{ }^{2}+\lambda_{3}{ }^{2}\right)}}
$$

7. Use the DTI software program to display pixel by pixel in each slice throughout both breasts a vector map showing the direction of the prime eigenvector, $\mathrm{v}_{1}$, and a color coded map with three colors indicating the main directions of $\mathrm{v}_{1}$ and overlay them on a T2 weighted image of the same slice (Figure 9). This step was performed using the free software gtk (http://gtk.org) for graphics.

8. Use the DTI software program to construct the diffusion tensor imaging parametric maps that display pixel by pixel in each slice throughout both breasts the values of all the diffusion tensor parameters $\lambda_{1}, \lambda_{2}, \lambda_{3}$ ADC $\lambda_{1}-\lambda_{3}$, and FA and overlay them on a T2 weighted image of the same slice (Figure 11). 

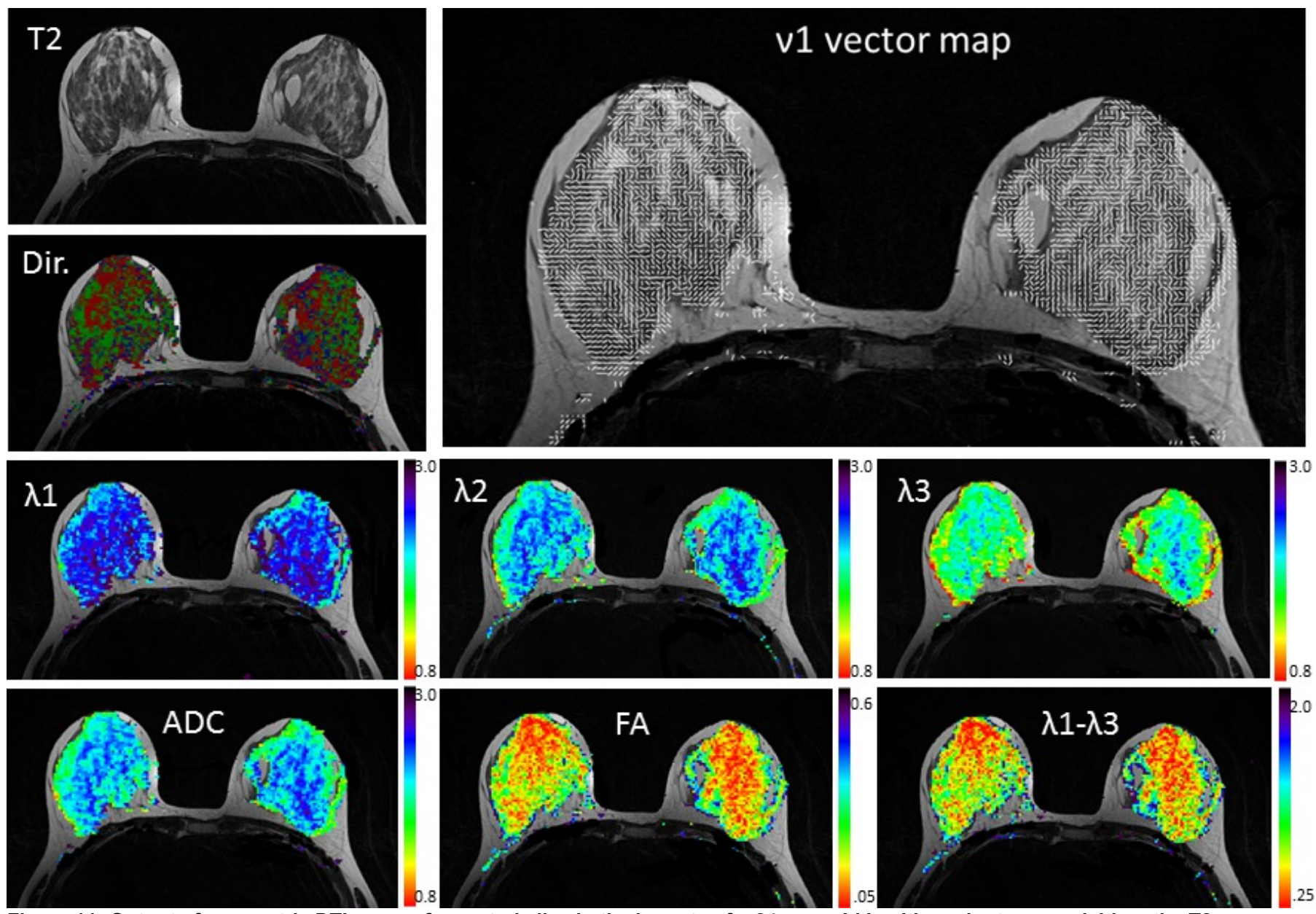

Figure 11: Output of parametric DTI maps of a central slice in the breasts of a 24 year old healthy volunteer overlaid on the T2

weighted image of the same slice. Dir. - Direction map with red indicating left to right, green indicating anterior to posterior and green indicating head to feet directions. Note that the vectors in the vector map are marked in white lines. All diffusion coefficients and the maximal anisotropy index are in units of $10^{-3} \mathrm{~mm}^{2} / \mathrm{sec}$. FA is unit-less. The scale bar in the vector map refers to a length of $20 \mathrm{~mm}$ and the scale bar in the T2 image refers to a length of $20 \mathrm{~mm}$ in all the remaining images.

3. Use the DTI software program to correct, if necessary, geometric distortions by loading the phase difference map obtained as described in step 2.6. Shift the range of the phase difference map in all pixels above the noise level in the gradient echo image to a range of $-\pi$ to $+\pi$ representing the phase shift $\Delta \phi(\mathrm{x}, \mathrm{y}, \mathrm{z})$ in radians (Figure 12).

1. Use the DTI software program to calculate the spatial shift in pixels according to:

$$
\Delta x=\frac{\Delta \phi(x, y, z)}{2 \pi \cdot \Delta T E \cdot B W p}
$$

$\triangle T E$ is the time difference between the two echoes equals $2.46 \times 10^{-3} \mathrm{msec}$ and $\mathrm{BWp}$ is the phase bandwidth in the experiment equals 13.2 Hz/pixel. Shift the pixels according to the field map and recalculate the diffusion tensor parameters (Figure 12). 

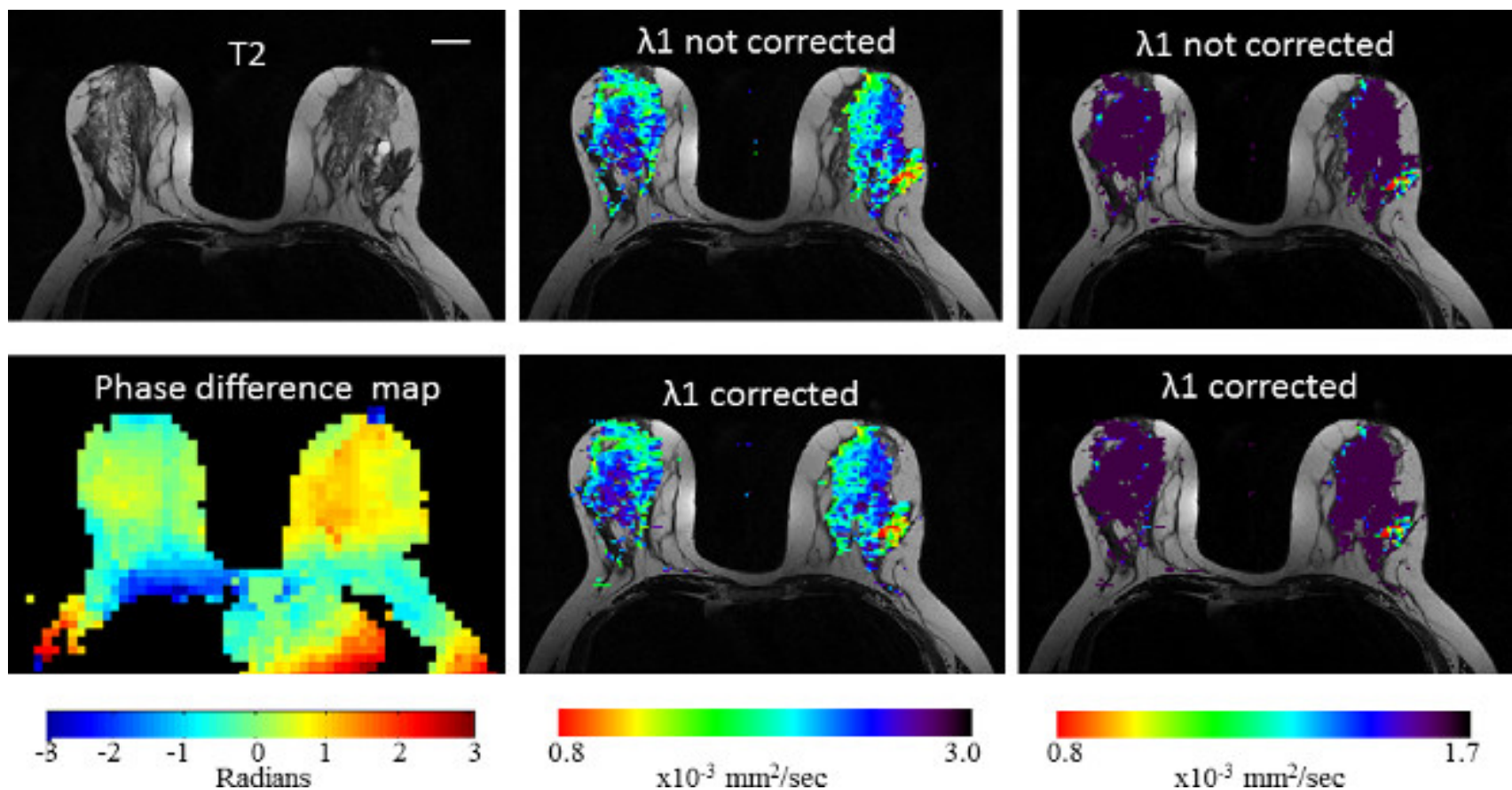

0.8

$\mathrm{x} 10^{-3} \mathrm{~mm}^{2} / \mathrm{sec}$
3.0
0.8

Figure 12: Example of geometric distortion correction using a phase difference map. The images were recorded from a 47 year old volunteer with invasive lobular carcinoma. In the first column on the left T2 weighted image and a phase difference map of a slice with cancer in the left breast are displayed. In the second and $3^{\text {rd }}$ columns $\lambda_{1}$ maps before correction are displayed in the first row and $\lambda_{1}$ maps after correction are displayed in the second row. The $\lambda_{1}$ maps are overlaid on the corresponding T2 weighted image. In the $2^{\text {nd }}$ column the full range of diffusion coefficients of water $(0.8-3.0) \times 10^{-3} \mathrm{~mm}^{2} / \mathrm{sec}$ is used for the color coded scale and in the $3^{\text {rd }}$ column a reduced range is used with a threshold of $1.7 \times 10^{-3} \mathrm{~mm}^{2} / \mathrm{sec}$ that emphasizes the contrast between the cancer in the left breast and the normal breast tissue. The scale bar in the T2 image refers to a length of $20 \mathrm{~mm}$ in all the images.

4. Perform image processing of the DCE-MRI datasets using a software that provides pixel by pixel maps of the kinetic parameters of the enhancement such as the 3TP image processing software ${ }^{17}$

\section{Representative Results}

The method was initially tested and demonstrated by scanning normal volunteers at various hormonal stages. Figure 11 demonstrated the parametric and vector maps obtained using our software of a central slice of a young healthy volunteer with relatively high fraction of fibroglandular tissue, as can be clearly seen on the T2 weighted image (all gray areas are fibroglandular tissue and bright areas are fat). The direction of the prime diffusion coefficient $\lambda_{1}$ is shown in the vector map v1 with a large fraction of pixels pointing towards the nipple. As expected the values of the diffusion tensor coefficients decline from $\lambda_{1}$ to $\lambda_{2}$ to $\lambda_{3}$. Using these three diffusion coefficients enabled calculation of the average diffusivity ADC, the fractional anisotropy (FA) and the maximal anisotropy, $\lambda_{1}-\lambda_{3}$. Notice in the maps of FA and $\lambda_{1}-\lambda_{3}$ the high congruence in the spatial distribution of the values of these two indices.

In post-menopausal women the breasts are usually less dense as shown in the example in Figure 13 for a 63 year old normal volunteer. The diffusion coefficients are lower in the elderly volunteer but the anisotropy indices are higher, possibly because of the lower diameter of the ducts and lobules and hence, higher restriction imposed on the diffusion in the directions orthogonal to the ductal walls. Another example demonstrating the sensitivity of the tensor to the size of the ducts is shown in the example of a lactating volunteer in Figure 14. Since milk is a colloid of fat globules within a water-based fluid that contains dissolved carbohydrates and protein aggregates with minerals, the viscosity of milk is higher than that of the normal water fluid in the ducts, and thus, the water diffusion coefficients of lactating breasts are lower than those of premenopausal women ${ }^{24}$. Furthermore, the ducts near the nipple are large and the restriction in the directions orthogonal to the ducts is lower than in the non-lactating breast, and consequently the anisotropy indices are lower as well. However in the lobular -posterior regions of the lactating breast the anisotropy is still high.

In patients with malignancy the main change in the diffusion tensor parameters is exhibited in a significant reduction in the three diffusion coefficients. The changes in $\lambda_{1}$ were found to provide the highest contrast to noise ratio ${ }^{19}$. This study included 68 patients with confirmed pathology including 33 patients diagnosed with invasive ductal carcinoma (IDC), 19 with ductal carcinoma in situ (DCIS), 13 with invasive lobular carcinoma (ILC) and 3 with other malignancies. Several patients had multi focal or multi centric breast cancer. The size of the cancers varied from 3 to $95 \mathrm{~mm}$ with median of $14 \mathrm{~mm}$ and interquartile range of 10 to $30 \mathrm{~mm}$. In all of these patients the detection rate of DCE and DTI were comparable. However, 5 cases that showed enhancement in DCE and were confirmed by pathology as cancers were not included in the DTI analysis due to technical problems in fatty breasts, primarily related to field inhomogeneity and insufficient fat suppression resulting in distortions and artifacts.

Figures 15, 16 and 17 demonstrate typical parametric maps of the two main diffusion parameters $\lambda_{1}$ and $\lambda_{1}-\lambda_{3}$ that are used for detecting breast malignancies. This figure shows, in addition to T2 weighted images, the results of DCE analysis using the 3TP color-coded method. As explained in the Introduction the presence of malignant cells in the ducts or lobules and their surrounding impede the diffusion in the extracellular 
compartment reducing significantly the diffusion coefficients. In addition, the anisotropy due to ductal structures is disappearing as the cancer cells spread in all directions in a chaotic way with no distinct direction. The FA is not an adequate parameter for breast cancer detection since the normalization of this parameter to the average diffusivity leads to high values of FA in cancers similar to those in normal tissue ${ }^{19}$. However, the maximal anisotropy does provide a means for detecting cancer (Figures 15-17). Nevertheless, because the connective fibrous tissue is also close to isotropic and exhibit low values of maximal anisotropy, this parameter is less specific than the diffusion coefficient $\lambda_{1}$ and acts as a secondary parameter to $\lambda_{1}$.

Figure 17 also demonstrates the ability of DTI to characterize response to neoadjuvant chemotherapy. In this example, the patient fully responded to the treatment (4 cycles of Adriamycin + Cycloxane -, followed by 4 cycles of Taxol). Indeed, the response to therapy caused a significant increase in the diffusion coefficients to values typical to normal breast tissue, suggesting the presence of reparative connective tissue that replaced the cancer cells. Similar results were obtained in 4 other patients that responded to therapy.
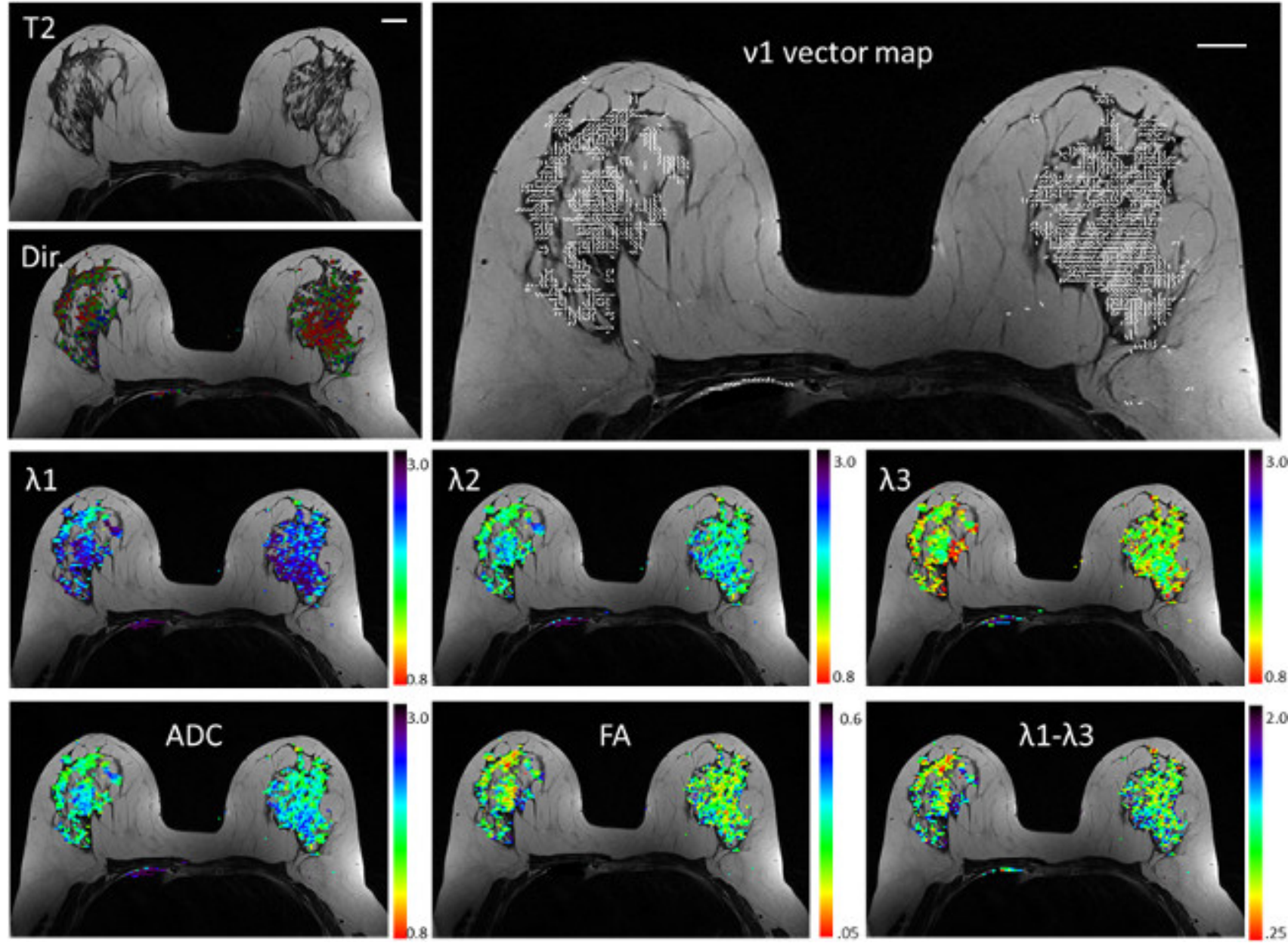

Figure 13: Output of parametric DTI maps of a central breast slice of a 63 years old healthy volunteer overlaid on the T2 weighted image of the same slice. Dir. - Direction map with red indicating left to right, green indicating anterior to posterior and green indicating head to feet directions. Note that the vectors are marked in white lines. All the diffusion coefficients and the maximal anisotropy index are in units of 1 $\times 10^{-3} \mathrm{~mm}^{2} / \mathrm{sec}$. FA is unit-less. The scale bar in the vector map refers to a length of $20 \mathrm{~mm}$ and the scale bar in the T2 image refers to a length of $20 \mathrm{~mm}$ in all the remaining images. 

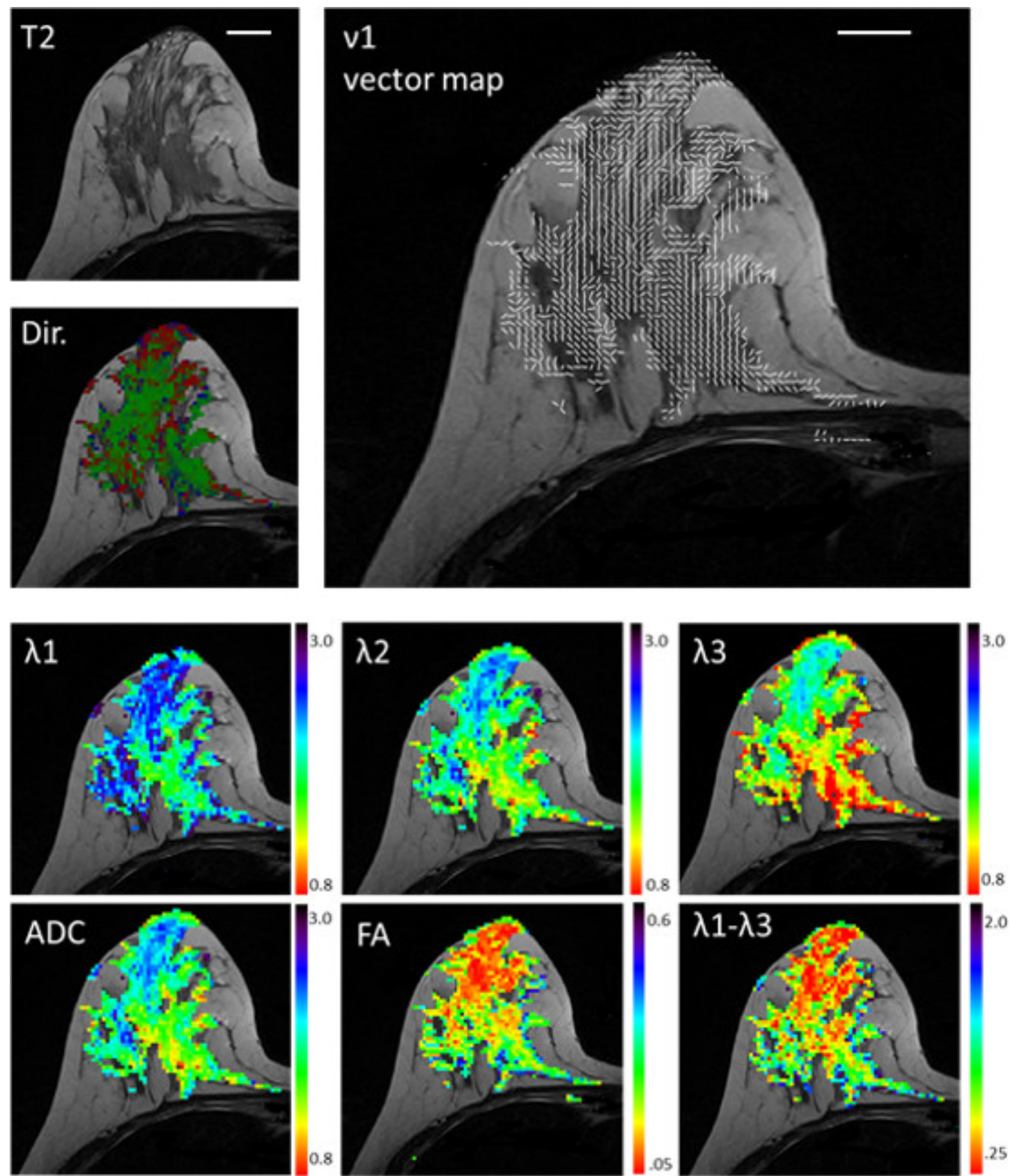

Figure 14: Output of parametric DTI maps of a central breast slice of a $\mathbf{4 0}$ years old lactating volunteer overlaid on the T2 weighted image of the same slice. Dir. - Direction map with red indicating left to right, green indicating anterior to posterior and green indicating head to feet directions. All the diffusion coefficients and the maximal anisotropy index are in units of $1 \times 10^{-3} \mathrm{~mm}^{2} / \mathrm{sec}$. FA is unit-less. Note in the direction map and vector map that most pixels are aligned towards the nipple. Also note the lower diffusion coefficients relative to the values in Figures 7 and 10 of healthy volunteers. The scale bar in the vector map refers to a length of $20 \mathrm{~mm}$ and the scale bar in the T2 image refers to a length of $20 \mathrm{~mm}$ in all the remaining images. 

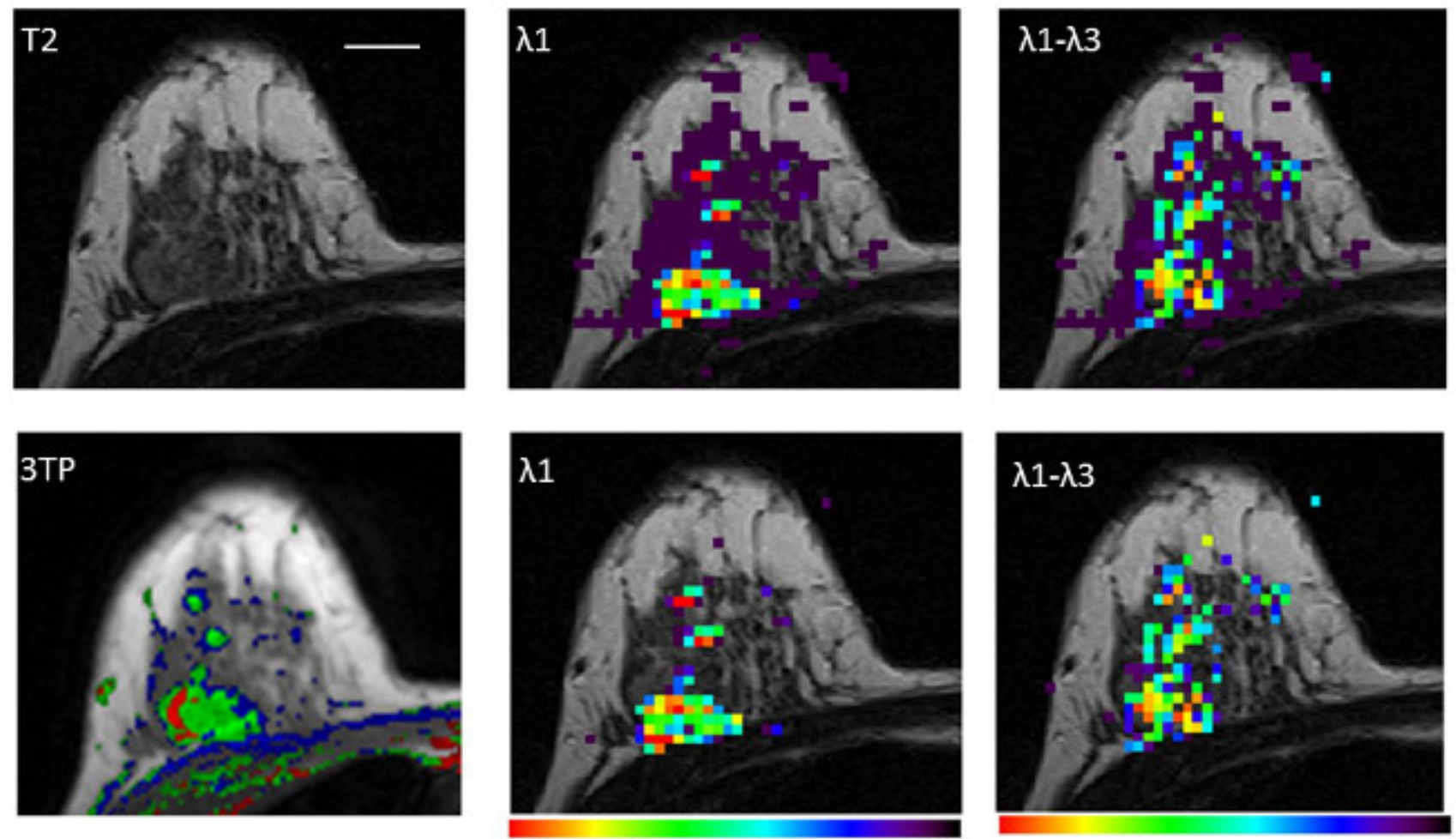

\section{8}

$\mathrm{x} 10^{-3} \mathrm{~mm}^{2} / \mathrm{sec}$

1.7

0.2

$\mathrm{x} 10^{-3} \mathrm{~mm}^{2} / \mathrm{sec}$

1.7

Figure 15: Parametric maps of $\lambda_{1}$ and $\lambda_{1}-\lambda_{3}$ in a 38 years old patient with multi focal invasive ductal carcinoma. In the $1^{\text {st }}$ raw the DTI parameters are presented with a threshold of $1.7 \times 10^{-3} \mathrm{~mm}^{2} / \mathrm{sec}$ for $\lambda_{1}$ and $0.6 \mathrm{~mm}^{2} / \mathrm{sec}$ for $\lambda_{1}-\lambda_{3}$ (all values above the thresholds are colored in purple). In the $2^{\text {nd }}$ raw, values above the threshold are not colored and show the underlying T2 weighted image. The figure also includes in the $1^{\text {st }}$ column on the left a T2 weighted image and a DCE parametric map, obtained by the 3TP method, of the same slice as the DTI parametric maps. The scale bar in the T2 image refers to a length of $20 \mathrm{~mm}$ in all the images. Note that the in-plane spatial resolution of the DCE images is approximately twice higher than that of DTI, however, there is a high visual congruence in the location and size of the 3TP and the $\lambda_{1}$ parametric maps.
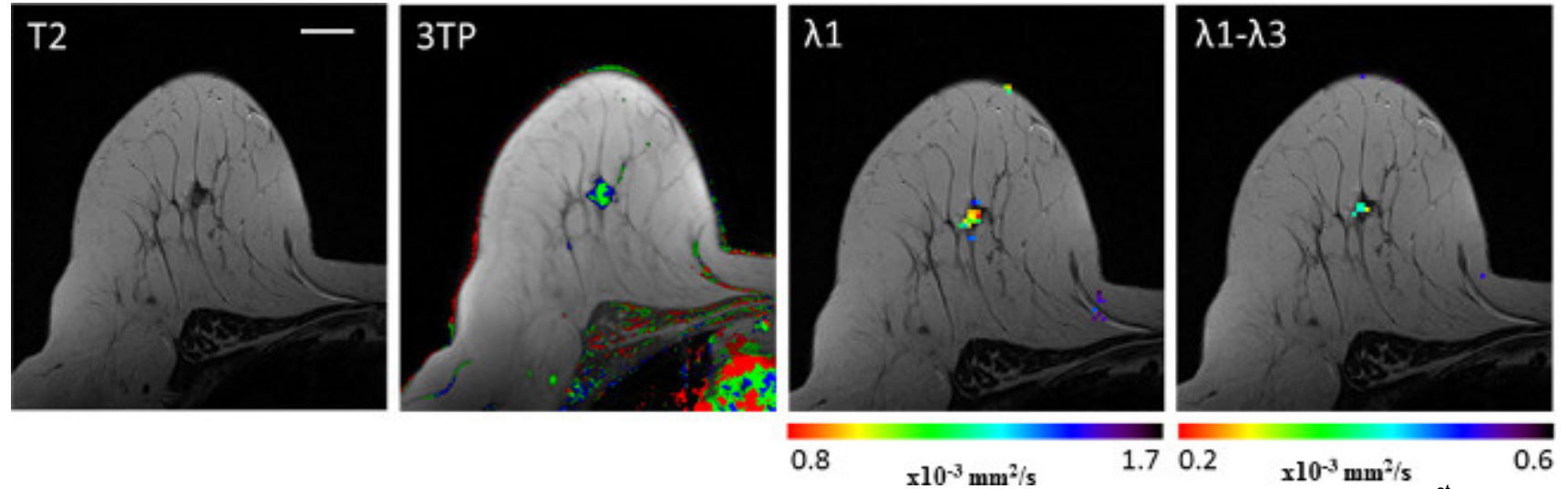

Figure 16: Parametric maps of $\lambda_{1}$ and $\lambda_{1}-\lambda_{3}$ in a 60 years old patient with low grade DCIS. The figure also includes in the $1^{\text {st }}$ column on the left a T2 weighted image and a DCE parametric map, obtained by the 3TP method, of the same slice as the DTI parametric maps. The scale bar in the T2 image refers to a length of $20 \mathrm{~mm}$ in all the images. Note the ability of DTI to detect cancer in a highly fatty breast. 

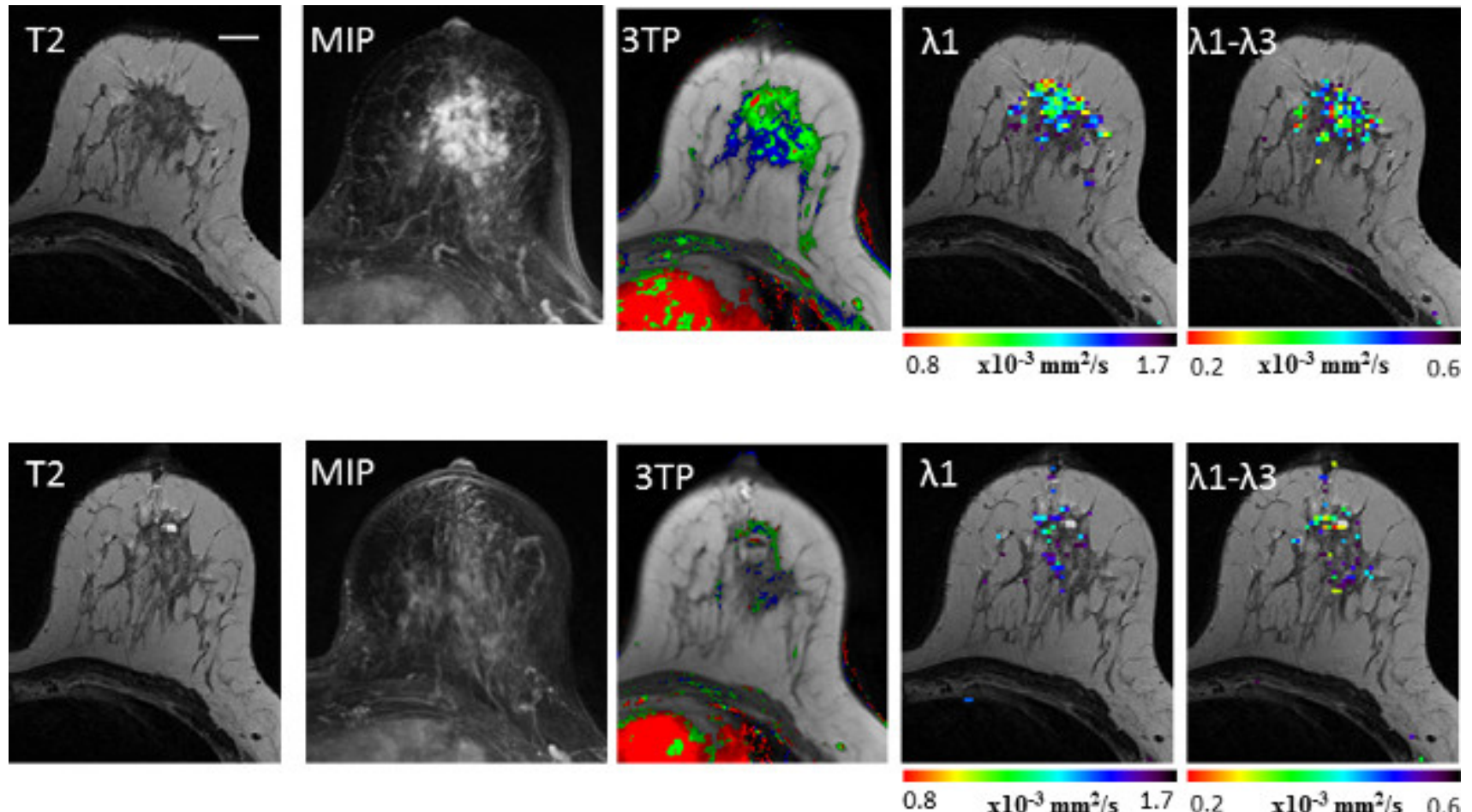

Figure 17: Parametric maps of $\lambda_{1}$ and $\lambda_{1}-\lambda_{3}$ in a 39 years old patient with invasive lobular carcinoma before and after neoadjuvant chemotherapy. The patient was scanned twice, once before therapy and once before surgery, after 4 cycles of Adryamicin + Cycloxan and 4 cycles of Taxol. Note the increase in $\lambda_{1}$ and in $\lambda_{1}-\lambda_{3}$ in cancer regions that responded to the treatment. The $1^{\text {st }}$ raw shows images obtained before therapy and the $2^{\text {nd }}$ raw shows images of approximately the same region as in the $1^{\text {st }}$ row, obtained before surgery. Before treatment cancer regions exhibited $\lambda_{1}$ and $\lambda_{1}-\lambda_{3}$ values below their threshold, and after treatment these two parameters increased to values above their threshold. The figure also includes in the $1^{\text {st }}$ column on the left T2 weighted images; in the $2^{\text {nd }}$ column Multiple Image Projection-MIP, obtained by subtracting the pre-contrast images from the 2 min post contrast images; in the $3^{\text {rd }}$ column DCE parametric maps obtained by the 3TP method. The scale bar in the T2 image refers to a length of $20 \mathrm{~mm}$ in all the images

\section{Discussion}

This work demonstrates the ability of DTI, scanned at $3 \mathrm{~T}$ at high spatial resolution $\left(\sim 8 \mathrm{~mm}^{3}\right)$, to measure in vivo the water diffusion tensor parameters in the entire fibroglandular tissue of both breasts. The ensemble of algorithms and software developed in the course of this study enabled analysis of the large DTI datasets and simultaneously view the diffusion parametric maps of the various diffusion coefficients, $\lambda_{1}, \lambda_{2}, \lambda_{3}$, ADC and anisotropy indices $\lambda_{1}-\lambda_{3}$, and FA of all breast slices. This work also emphasizes the potential of the various diffusion coefficients and anisotropy indices to detect and diagnose breast cancer. The findings indicated that the prime diffusion coefficient, $\lambda_{1}$, is the principal parameter for detecting breast cancer, with a substantial ability to differentiate malignant from normal breast tissue. A secondary independent parameter, with high sensitivity but much lower specificity than $\lambda_{1}$, is the maximum anisotropy index which acts to confirm the detection by the prime diffusion coefficient.

The results also demonstrated the ability of MRI to measure in vivo the directional water diffusion and quantify the anisotropy of the diffusion in the entire ductal/glandular system in both breasts. In order to ensure a uniform precision profile of the anisotropic measurements 30 diffusion gradient directions were applied. The selection of high echo-time of $120 \mathrm{msec}$, and the optimization of the spatial resolution revealed the restriction of the water diffusion in the ducts. The average size of normal ducts was reported to be $90 \mu \mathrm{m}$, with $\sim 70 \%$ in the range of $40-100$ $\mu \mathrm{m}^{36}$. According to Einstein equation the mean displacement of free water diffusion, $x=(6 \mathrm{Dt})^{1 / 2}$, where $\mathrm{D}$ is the free water diffusion coefficient and $t$ the diffusion time. In our experiment $\mathrm{x}$ is approximately $25 \mu \mathrm{m}$, and hence, only a fraction of the ductal water molecules are restricted by the mammary ductal walls, leading to the relative low FA values in the breast (upper values $\sim 0.3$ ) as compared to the values found in brain white matter $(\geq 0.5)$. In Sir Astley Cooper studies ${ }^{25}$ it was revealed that human breast tissue is organized into separate lobes, each composed of a ductal tree. Preliminary ultrasound studies of a lactating breast ${ }^{37}$ were attempted, as well as detection of sectional ductal structures using second-order shape measurements ${ }^{38}$. However, no imaging method has succeeded thus far revealing the entire ductal systems in both breasts in vivo. The algorithm for obtaining the entire $3 \mathrm{D}$ ductal tree system from the DTI results is still under development but the vector maps clearly exhibit the potential to reveal the detailed and highly diversified anatomy of the mammary trees. Recently an initial attempt of a full 3D tracking of the breast based on our DTI datasets was reported ${ }^{39}$.

In the course of this work technical limitations have been noticed due to inefficient fat suppression and EPI distortions. Fat suppression was achieved by a fat saturation sequence (FAT-SAT) which was usually efficient in dense breasts. However, in fatty breasts Spectral Selection Attenuated Inversion Recovery (SPAIR), which is more efficient and less sensitive to B1 inhomogeneity, was applied. The EPI based diffusion protocols had additional limitations due to gradient induced eddy currents, $B_{0}$ field inhomogeneity and susceptibility differences ${ }^{40,41}$. These limitations were minimized by using iterative automatic and manual shimming on the water and fat signals and choosing the lower possible echo spacing. In addition, a post processing correction of geometrical distortions was occasionally applied as described in the Protocol. Overall, in the 
case of dense breasts with a strong water signal it was possible to overcome the technical limitations; however, 5 cases in this study with highly fatty breasts could not be analyzed due to the above limitations.

In summary, a protocol and image processing tools for breast diffusion tensor MRI have been developed. This completely safe, rapid, and noninvasive methodology precisely dissects breast architecture and can facilitate detection of breast cancer in the clinic. The prime diffusion coefficient, $\lambda_{1}$, and the maximal anisotropy index, $\lambda_{1}-\lambda_{3}$, were found to serve as two independent diffusion parameters for detecting cancer. Clinical studies of breast cancer patients showed that the detection efficiency with these two parameters is comparable to that of DCE MRI. Thus, the emerging of this method from basic understanding of the breast architecture and of cancer development and its reliance on revealing quantifiable significant changes, as well as its being a safe and fast method make it a valuable tool for investigating various developmental aspects involving ductal proliferation, and for testing its clinical impact for breast cancer screening in large scale prospective trials.

\section{Disclosures}

The authors have nothing to disclose.

\section{Acknowledgements}

We would like to thank Mr. Nachum Stern and Ms. Fanny Attar for their excellent technical assistance. H.D. holds the Fred and Andrea Fallek Chair for Breast Cancer Research.

\section{References}

1. Turnbull, L.W. Dynamic contrast-enhanced MRI in the diagnosis and management of breast cancer. NMR Biomed. 22 (1), 28-39, doi: 10.1002/nbm.1273 (2009).

2. Sardanelli, F., et al. Magnetic resonance imaging of the breast: recommendations from the EUSOMA working group. Eur J Cancer. 46 (8), 1296-1316, doi: 10.1016/j.ejca.2010.02.015 (2010).

3. Lehman, C.D., et al. Accuracy and interpretation time of computer-aided detection among novice and experienced breast MRI readers. AJR Am J Roentgenol. 200 (6), W683-689, doi: 10.2214/AJR.11.8394 (2013).

4. Riedl, C.C., et al. Magnetic resonance imaging of the breast improves detection of invasive cancer, preinvasive cancer, and premalignant lesions during surveillance of women at high risk for breast cancer. Clin Cancer Res. 13 (20), 6144-6152 (2007).

5. Heywang-Köbrunner, S.H., Hacker, A., Sedlacek, S. Magnetic resonance imaging: the evolution of breast imaging. Breast. 22 Suppl 2 , S77-82, doi:10.1016/j.breast.2013.07.014 (2013).

6. Peters, N.H., et al. Meta-analysis of MR imaging in the diagnosis of breast lesions. Radiology. 246 (1),116-24 (2008).

7. Warner, E., et al. Systematic review: using magnetic resonance imaging to screen women at high risk for breast cancer. Ann Intern Med. 148 (9), 671-9 (2008).

8. Benndorf, M., et al. Breast MRI as an adjunct to mammography: Does it really suffer from low specificity? A retrospective analysis stratified by mammographic BI-RADS classes. Acta Radiol. 51 (7), 715-21, doi: 10.3109/02841851.2010.497164. (2010).

9. Thomassin-Naggara, I., De Bazelaire, C., Chopier, J., Bazot, M., Marsault, C. Trop I Diffusion-weighted MR imaging of the breast: advantages and pitfalls. Eur J Radiol. 82 (3), 435-443, doi: 10.1016/j.ejrad.2012.03.002 (2013).

10. Bogner, W., et al. Diffusion-weighted MR for differentiation of breast lesions at $3.0 \mathrm{~T}$ : how does selection of diffusion protocols affect diagnosis? Radiology. 253 (2), 341-351, doi: 10.1148/radiol.2532081718 (2009).

11. Partridge, S.C. et al. Diffusion tensor imaging of the breast: preliminary clinical findings [abstr]. In Proceedings of the Fourteenth Meeting of the International Society for Magnetic Resonance in Medicine. Berkeley, CA: International Society for Magnetic Resonance in Medicine 2902 (2006).

12. Partridge, S.C., et al. Diffusion tensor MRI: preliminary anisotropy measures and mapping of breast tumors. J Magn Reson Imaging. 31 (2), 339-47, doi: 10.1002/jmri.22045 (2010).

13. Partridge, S.C., et al. Diffusion tensor magnetic resonance imaging of the normal breast. Magn Reson Imaging. 28 (3), $320-8$, doi: 10.1016/ j.mri.2009.10.003 (2010).

14. Baltzer, P.A., et al. Diffusion tensor magnetic resonance imaging of the breast: a pilot study. Eur Radiol. 21 (1), 1-10. doi: 10.1007/ s00330-010-1901-9 (2011).

15. Wang, Y., et al. Optimization of the parameters for diffusion tensor magnetic resonance imaging data acquisition for breast fiber tractography at 1.5 T. Clin Breast Cancer. 14(1), 61-7, doi: 10.1016/j.clbc.2013.09.002 (2014).

16. Eyal, E., and Degani, H. Method and apparatus for ductal tube tracking imaging for breast cancer and diagnosis and product. U.S. Patent provisional application 61/044,697, Granted U.S. Patent 8, 526,69 (2008).

17. Eyal, E., et al. Novel MRI method for breast cancer detection based on diffusion tensor tracking of the ductal trees [abstr]. In: Proceedings of the Eighteenth Meeting of the International Society for Magnetic Resonance in Medicine. Berkeley, CA: International Society for Magnetic Resonance in Medicine, 362 (2010).

18. Furman-Haran, E., et al. Breast cancer detection and diagnosis based on diffusion tensor imaging [abstr]. In: Proceedings of the Nineteenth Meeting of the International Society for Magnetic Resonance in Medicine. Berkeley, CA: International Society for Magnetic Resonance in Medicine, 515 (2011).

19. Eyal, E., et al. Parametric diffusion tensor imaging of the breast. Invest Radiol. 47 (5), 284-291, doi: 10.1097/RLI.0b013e3182438e5d (2012).

20. Tagliafico, A., et al.,Diffusion tensor magnetic resonance imaging of the normal breast: reproducibility of DTI-derived fractional anisotropy and apparent diffusion coefficient at 3.0 T. Radiol Med. 117 (6), 992-1003 (2012).

21. Cakir, O., et al., Comparison of the diagnostic performances of diffusion parameters in diffusion weighted imaging and diffusion tensor imaging of breast lesions.Eur J Radiol. 82 (12), e801-6, doi: 10.1016/j.ejrad.2013.09.001 (2013).

22. Tsougos, I. et al. The contribution of diffusion tensor imaging and magnetic resonance spectroscopy for the differentiation of breast lesions at 3T. Acta Radiol. 55 (1), 14-2, doi: 10.1177/0284185113492152.T (2014). 
23. Wiederer, Pazahr S., Leo, C., Nanz, D., Boss, A. Quantitative breast MRI: 2D histogram analysis of diffusion tensor parameters in normal tissue. Magn Reson Mater Phy. 27, 185-193, doi 10.1007/s10334-013-0400-9 (2014).

24. Nissan, N., Furman-Haran E., Shapiro-Feinberg M., Grobgeld D., Degani H. Diffusion-tensor MR imaging of the breast: hormonal regulation. Radiology. 271 (3), 672-80, doi: 10.1148/radiol.14132084 (2014).

25. Cooper, A.P. On the Anatomy of the breast. Longman, Orme, Green, Browne and Longmans, London. http://jdc.jefferson.edu/cooper (1840).

26. Ohtake, T., et al. Computer-assisted complete three-dimensional reconstruction of the mammary ductal/lobular systems: implications of ductal anastomoses for breast-conserving surgery. Cancer. 91 (12), 2263-2272 (2001).

27. Going, J.J. and Moffat, D.F. Escaping from Flatland: clinical and biological aspects of human mammary duct anatomy in three dimensions. $J$ Pathol. 203 (1), 538-544 (2004).

28. Hancu, I., Govenkar, A., Lenkinski, R.E., Lee, S.K. On shimming approaches in 3T breast MRI. Magn Reson Med. 69 (3), 862-867, doi: 10.1002/mrm.24307 (2013).

29. Basser, P.J., Jones, D.K. Diffusion-tensor MRI: theory, experimental design and data analysis - a technical review. NMR Biomed. 15 (7-8), 456-467 (2002).

30. Reese, T.G., Heid, O., Weisskoff, R.M., Wedeen, V.J. Reduction of eddy-current-induced distortion in diffusion MRI using a twice-refocused spin echo. Magn Reson Med. 49 (1), 1771-1782 (2003).

31. Jezzard, P., Balaban, R. Correction for geometric distortion in echo planar images from B0 field variations. Magn Reson Med. 34 (1), $65-73$ (1995).

32. Kelcz, F., Furman-Haran, E., Grobgeld, D., Degani, H. Clinical testing of high-spatial resolution parametric contrast-enhanced MR imaging of the breast. AJR Am J Roentgenol. 179 (6), 1485-1492 (2002).

33. Le Bihan, D., et al. Diffusion tensor imaging: concepts and applications. J Magn Reson Imaging. 13 (4), $534-546$ (2001).

34. Marquardt, D. An Algorithm for Least-Squares Estimation of Nonlinear Parameter. SIAM Journal on Applied Mathematics. 11 (2): 431-441, doi: 10.110111030 (1963).

35. Jolliffe, I.T. Principal Component Analysis: A Beginner's Guide — I. Introduction and application. Weather. 45 (10), 375-382, doi: 10.1002/ j.1477-8696.1990.tb05558.x (1990).

36. Mayr, N.A., Staples, J.J., Robinson, R.A., Vanmetre, J.E., Hussey, D.H. Morphometric studies in intraductal breast carcinoma using computerized image analysis. Cancer. 67 (11), 2805-2812 (1991).

37. Ramsay, D.T., Kent, J.C., Hartmann, R.A., Hartmann, P.E. Anatomy of the lactating human breast redefined with ultrasound imaging. J Anat 206 (6), 525-534 (2005).

38. Gooding, M.J., Mellor, M., Shipley, J.A., Broadbent, K.A., Goddard, D.A. Automatic mammary duct detection in 3D ultrasound. Med Image Comput Comput Assist Interv. 8 (1), 434-441 (2005).

39. Reisert, M.M., Eyal, E., Grobgeld, D., Degani, H., Hennig, J. Diffusion tensor based reconstruction of the ductal tree [abstr]. In: Proceedings of the Nineteenth Meeting of the International Society for Magnetic Resonance in Medicine. Berkeley, Calif: International Society for Magnetic Resonance in Medicine. 3649 (2011).

40. Jezzard, P., Clare, S. Sources of distortion in functional MRI data. Hum Brain Mapp. 8 (2-3), $80-85$ (1999).

41. Jones, D.K., Cercignani, M. Twenty-five pitfalls in the analysis of diffusion MRI data. NMR Biomed. 23 (7), 803-820, doi: 10.1002/nbm.1543 (2010). 